\title{
Correction to: Clinical use of physiological lesion assessment using pressure guidewires: an expert consensus document of the Japanese Association of Cardiovascular Intervention and Therapeutics
}

\author{
Yoshiaki Kawase ${ }^{1}$ (D) Hitoshi Matsuo ${ }^{1} \cdot$ Takashi Akasaka $^{2} \cdot$ Yasutsugu Shiono $^{2} \cdot$ Nobuhiro Tanaka $^{3} \cdot$ Tetsuya Amano $^{4}$. \\ Ken Kozuma ${ }^{5} \cdot$ Masato Nakamura $^{6} \cdot$ Hiroyoshi Yokoi $^{7} \cdot$ Yoshio Kobayashi $^{8} \cdot$ Yuji lkari $^{9}$
}

Published online: 7 July 2020

(c) Japanese Association of Cardiovascular Intervention and Therapeutics 2020

\section{Correction to: \\ Cardiovascular Intervention and Therapeutics \\ (2019) 34:85-96 \\ https://doi.org/10.1007/s12928-018-0559-0}

In the original publication of the article, the affiliations of the author group were published incorrectly. The correct affiliations of author group are given in this Correction.

Publisher's Note Springer Nature remains neutral with regard to jurisdictional claims in published maps and institutional affiliations.

The original article can be found online at https://doi.org/10.1007/ s12928-018-0559-0.

Yoshiaki Kawase

ykawase@ heart-center.or.jp

1 Department of Cardiovascular Medicine, Gifu Heart Center, Gifu, Japan

2 Department of Cardiovascular Medicine, Wakayama Medical University, Wakayama, Japan

3 Department of Cardiology, Tokyo Medical University Hachioji Medical Center, Tokyo, Japan

4 Department of Cardiology, Aichi Medical University, Aichi, Japan

5 Department of Cardiology, Teikyo University Hospital, Tokyo, Japan

6 Division of Cardiovascular Medicine, Ohashi Medical Center, Toho University School of Medicine, Tokyo, Japan

7 Department of Cardiology, Fukuoka Sanno Hospital, Fukuoka, Japan

8 Department of Cardiovascular Medicine, Chiba University Graduate School of Medicine, Chiba, Japan

9 Department of Cardiology, Tokai University Hospital, Isehara, Japan 